\title{
Development of a mnemonic discrimination task using naturalistic stimuli with applications to aging and preclinical Alzheimer's disease
}

\author{
Stephanie L. Leal, ${ }^{1}$ Lorena A. Ferguson, ${ }^{1}$ Theresa M. Harrison, ${ }^{1}$ and William J. Jagust ${ }^{1,2}$ \\ ${ }^{1}$ Helen Wills Neuroscience Institute, University of California, Berkeley, Berkeley, California 94720, USA; ${ }^{2}$ Molecular Biophysics \\ and Integrated Bioimaging, Lawrence Berkeley National Laboratory, Berkeley, California 94720, USA
}

\begin{abstract}
Most tasks test memory within the same day, however, most forgetting occurs after $24 \mathrm{~h}$. Further, testing memory for simple words or objects does not mimic real-world memory experiences. We designed a memory task showing participants video clips of everyday kinds of experiences, including positive, negative, and neutral stimuli, and tested memory immediately and $24 \mathrm{~h}$ later. During the memory test, we included repeated and similar stimuli to tax both target recognition and lure discrimination ability. Participants' memory was worse after $24 \mathrm{~h}$, especially the ability to discriminate similar stimuli. Emotional videos were better remembered when tested immediately, however, after $24 \mathrm{~h}$ we find gist versus detail trade-offs in emotional forgetting. We also applied this paradigm to a sample of cognitively normal older adults that also underwent amyloid and tau PET imaging. We found that older adults performed worse on the task compared to young adults. While both young and older adults showed similar patterns of forgetting of repeated emotional and neutral clips, older adults showed preserved neutral compared to emotional discrimination after $24 \mathrm{~h}$. Further, lure discrimination performance correlated with medial temporal lobe tau in older adults with preclinical Alzheimer's disease. These results suggest factors such as time between encoding and retrieval, emotion, and similarity influence memory performance and should be considered when examining memory performance for an accurate picture of memory function and dysfunction.
\end{abstract}

Episodic memory, or memory for events in our lives, is an important facet of cognition that allows us to remember our past to influence future decisions (Tulving 2002). One critical component of memory is its counterpart forgetting, which is a necessary feature of memory to avoid catastrophic interference and overload (Wixted 2004). Initially, memories are labile and subject to change and forgetting, but over time become more stable (i.e., become consolidated) (McGaugh 2000; Nader et al. 2000; Wixted 2004). Many memory tasks developed in the laboratory test memory within the same day and examine how much information is remembered. However, the amount of forgetting over time can be an important memory measure to consider since this is often the type of memory problem people struggle with in natural settings.

The hippocampus and surrounding cortical regions, or the medial temporal lobe (MTL), play a key role in episodic memory (Squire et al. 2004) and are involved in memory consolidation (McGaugh 2000). However, these regions are not likely to be the locus of the memory, but rather an index of the memory to link the association neocortex at the time of memory recall (Teyler and DiScenna 1986). Computational models propose that the hippocampus performs two key computations: pattern separation and pattern completion (Yassa and Stark 2011). Pattern separation is the process of reducing interference among similar inputs by using nonoverlapping representations and relies on the hippocampal dentate gyrus (DG), while pattern completion retrieves previously stored information given partial or degraded cues (Marr 1971; Treves and Rolls 1994).

Discrimination tasks have been designed to tax hippocampal pattern separation by including test stimuli that are similar but not identical to studied items (Stark et al. 2013; Leal et al. 2014; Reagh

\section{Corresponding author: stephanieleal@berkeley.edu}

Article is online at http://www.learnmem.org/cgi/doi/10.1101/lm.048967.118. et al. 2014; Roberts et al. 2014). Tasks include word (Ly et al. 2013), object (Stark et al. 2013), or scene (Leal et al. 2014) stimuli and typically test memory on the same day. While these tasks use wellcontrolled stimuli, they often lack contextual information that exists in natural settings. Creating a more ecologically valid memory task that captures the complexity of everyday experiences can provide a more accurate measure of memory retention and loss. Ideally, tasks would capture the episodic memory elements of "what," "where," "when," and "why" in order to provide an accurate representation of a memory.

The significance of a memory (i.e., "why") when remembering an experience is often overlooked in traditional memory tasks. Participants are shown either largely neutral stimuli or stimuli that contain emotional components but with unmeasured relationships to memory performance. Previous work has shown that emotional arousal and valence of an event influences remembering and forgetting (McGaugh 2002, 2004; Kensinger 2009; Leal et al. 2014). Although memory for emotional events is often preserved (McGaugh 2004), this is not always the case for all types of information (Brown and Kulik 1977; Kensinger 2009; Leal et al. 2014). Gist versus detail trade-offs often occur when examining emotional versus neutral memory (Kensinger et al. 2007; Leal et al. 2014), where the emotional gist is retained at the expense of emotional details. This emotional gist versus detail trade-off has been previously examined via central versus peripheral components of an emotional event (Loftus et al. 1987; Adolphs et al. 2005; Kensinger et al. 2007) or more recently via target recognition versus

2019 Leal et al. This article is distributed exclusively by Cold Spring Harbor Laboratory Press for the first 12 months after the full-issue publication date (see http://learnmem.cshlp.org/site/misc/terms.xhtml). After 12 months, it is available under a Creative Commons License (Attribution-NonCommercial 4.0 International), as described at http://creativecommons.org/licenses/by-nc/4.0/. 
lure discrimination differences (Leal et al. 2014). This highlights the complexity of memory; contextual elements such as emotion play a crucial role in memory consolidation.

One population that shows deficits in episodic memory performance and the ability to discriminate highly overlapping experiences are older adults (Robitsek et al. 2008; Yassa et al. 2011b; Leal and Yassa 2015). Disambiguating similar experiences and overcoming interference is a critical feature of episodic memory (Marr 1971; Shapiro and Olton 1994) and increased susceptibility to interference has been found in aging across species (Wilson et al. 2006; Yassa et al. 2011a,b). Tasks have found age-related reductions in the ability to discriminate object (Toner et al. 2009; Ryan et al. 2012; Stark et al. 2013; Reagh et al. 2016), spatial (Gallagher et al. 1993; Wilson et al. 2004; Reagh et al. 2014), and temporal domains (Roberts et al. 2014) of memory. In aging, memory consolidation is typically impaired (Ward et al. 1999), although the degree of forgetting depends on the length of the delay, the type of information being remembered, and other factors that influence how memories are stored (Park et al. 1988; Ward et al. 1999; Davis et al. 2003; Leal and Yassa 2014). The MTL is also the first region to be affected by tau pathology (Braak and Braak 1996; Khan et al. 2014; Yassa 2014), which is ubiquitous in those over age 75 and is linked to episodic memory deficits in aging and Alzheimer's disease (AD) (Maass et al. 2018).

In the current study, we developed a more naturalistic memory task (Fig. 1) using dynamic video stimuli that featured everyday kinds of experiences (e.g., picking up medications, cleaning the kitchen, going to the grocery store) as well as experiences with more emotional context (i.e., getting into a car crash, baby learning to walk, sitting in traffic). Participants watched 10 -sec video clips to create a contextually rich encoding environment and were then tested on their memory of scenes using still images from the video clips immediately ( $\sim 5$ min after encoding) and 24 $\mathrm{h}$ later. We tested the specificity of memory by including similar lure stimuli (discrimination) and repeated target stimuli (recognition). We tested participants' memory immediately and $24 \mathrm{~h}$ later to examine changes in memory over time, which is important giv-

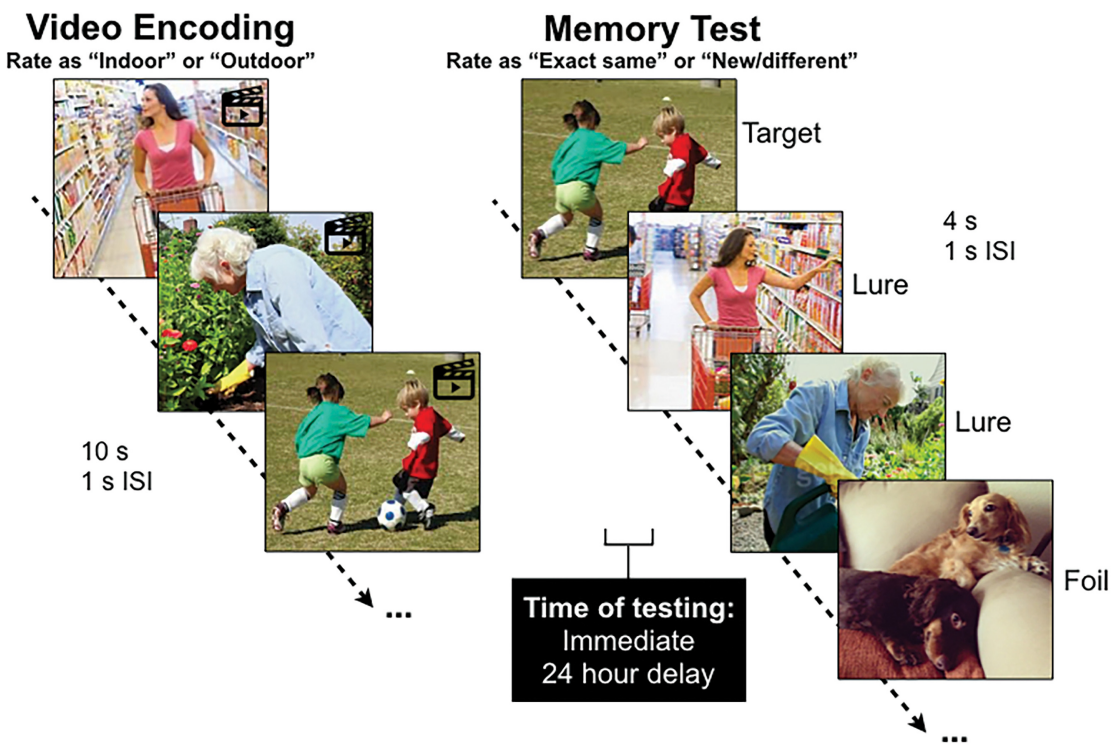

Figure 1. Video mnemonic discrimination task. Participants are shown $10 \mathrm{sec}$ video clips during encoding, in which they are asked to rate whether they take place more indoors or outdoors. Participants are then tested on their memory for scenes (shown for $4 \mathrm{sec}$ ) from the videos either immediately ( $\sim \mathrm{min}$ after encoding) and $24 \mathrm{~h}$ later. Scenes are comprised of the exact same scenes from the videos (targets), scenes that are similar but not exactly the same from the videos (lures), and scenes that were not shown in any of the videos (foils). en that most forgetting occurs after $24 \mathrm{~h}$ (Murre and Dros 2015). Finally, we tested the impact emotion has on memory by including emotional and neutral stimuli. We then applied this task to a sample of cognitively normal older adults to investigate age-related memory impairments. We hypothesized that (1) there would be increased forgetting over time, (2) target recognition (gist) would be better compared to lure discrimination (detail), (3) gist versus detail trade-offs may exist, where emotional gist may be preserved over time while emotional details may be impaired, and (4) older adults would show memory impairments compared to young adults and may show altered processing of emotional versus neutral stimuli.

\section{Results}

\section{Worse memory over $24 \mathrm{~h}$, especially when discriminating similar stimuli}

Thirty-seven young adults (mean age $=23,27 \%$ male) performed the video mnemonic discrimination task, with recognition testing immediately and $24 \mathrm{~h}$ later. We examined target recognition to measure more gist-like memory and lure discrimination to measure more detail-oriented memory, which is thought to tax hippocampal pattern separation (see Materials and Methods for more details). We performed a pairwise $t$-test within-subjects to compare performance immediately versus $24 \mathrm{~h}$ later. As expected, we found that both target recognition $\left(\mathrm{d}^{\prime}\right)\left(t_{(36)}=8.95, P<0.001\right.$; Fig. 2A) and lure discrimination $\left(\mathrm{d}^{\prime}\right)\left(t_{(36)}=7.27, P<0.001\right.$; Fig. $\left.2 \mathrm{~B}\right)$ of the scenes from the videos were worse after $24 \mathrm{~h}$ compared to immediate testing. We compared target recognition to lure discrimination using a repeated-measures ANOVA with time and memory type as withinsubjects factors. We found a significant main effect of time $\left(F_{(1,36)}=\right.$ $82.64, P<0.001)$, where memory was worse after a $24 \mathrm{~h}$ delay. We found a significant main effect of memory type $\left(F_{(1,36)}=\right.$ $728.17, P<0.001)$, where lure discrimination was worse compared to target recognition. There was a significant interaction between time and memory type $\left(F_{(1,36)}=12.41, P=0.001\right)$, such that the difference between target recognition and lure discrimination was greater immediately versus $24 \mathrm{~h}$ later. Participants' individual performance over time is shown in Figure 2C,D.

\section{Emotional memory is better immediately but gist versus detail trade-offs exist after $24 \mathrm{~h}$}

Next, we examined whether the emotional significance of the video stimuli affected memory performance immediately and $24 \mathrm{~h}$ later. We divided the stimuli into positive, negative, and neutral videos and conducted a repeated-measures ANOVA with emotion and time as withinsubjects factors. For target recognition, we found a significant main effect of emotion $\left(F_{(2,72)}=16.48, P<0.001\right.$; Fig. $\left.3 \mathrm{~A}\right)$, where emotional targets (both negative and positive) were better remembered compared to neutral targets (post-hoc Scheffé: $\left.F_{(1,36)}=26.08, P<0.001\right)$. We found a significant main effect of time $\left(F_{(1,36)}=\right.$ $118.53, P<0.001)$, where target recognition was better immediately compared to $24 \mathrm{~h}$ later. We also found a significant interaction between emotion and time $\left(F_{(2,72)}=5.85, P=0.004\right)$ such that emotional targets (both negative and positive) 
A
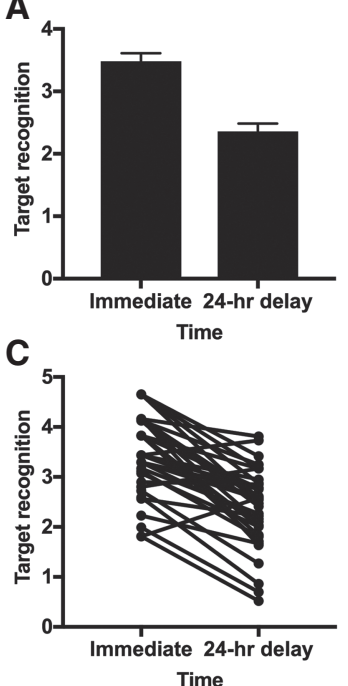

B

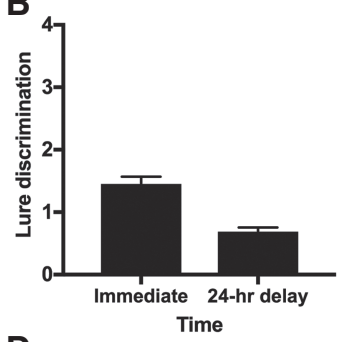

D

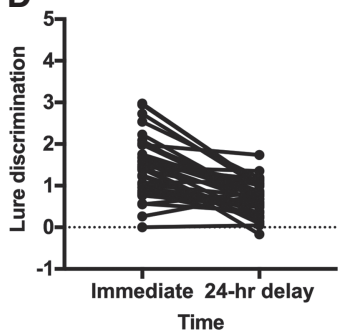

Figure 2. Young adult target recognition and lure discrimination performance immediately and $24 \mathrm{~h}$ later. $(A)$ Target recognition was better immediately compared to $24 \mathrm{~h}$ later, $(B)$ lure discrimination was better immediately compared to $24 \mathrm{~h}$ later, $(C)$ individual subject slopes for target recognition showing forgetting over a 24-h delay, $(D)$ individual subject slopes for lure discrimination showing forgetting over a 24-h delay.

were better remembered immediately compared to neutral targets while $24 \mathrm{~h}$ later only the negative stimuli were better remembered compared to neutral or positive targets (post-hoc Scheffé: $F_{(1,36)}=$ $7.89, P=0.008)$. This suggests a preservation of emotional negative gist information over time.

For lure discrimination, we found a significant main effect of emotion $\left(F_{(2,72)}=10.67, P<0.001\right.$; Fig. 3B), where emotional lures (both negative and positive) were better discriminated compared to neutral lures (post-hoc Scheffé: $F_{(1,36)}=21.21, P<0.001$ ). We found a significant main effect of time $\left(F_{(1,36)}=75.26, P<0.001\right)$, where lure discrimination was better immediately compared to $24 \mathrm{~h}$ later. We also found a significant interaction between emotion and time $\left(F_{(2,72)}=9.85, P<0.001\right)$ such that emotional lure discrimination was better immediately compared to neutral lure discrimination, however this difference was no longer evident after $24 \mathrm{~h}$ (post-hoc Scheffé: $\mathrm{F}_{(1,36)}=33.40, \quad P<0.001$ ). Thus, memory for highly detailed emotional stimuli is being degraded over time while neutral stimuli are subject to less forgetting over time.

Next, we calculated the proportion of stimuli forgotten over time by subtracting delayed from immediate performance and dividing by immediate performance to get a measure of forgetting on an individual subject basis [(Delayed - Immediate)/Immediate].

We conducted a repeated-measures ANOVA with emotion (negative, neutral, and positive) and memory type (target recognition/ gist vs. lure discrimination/detail) to determine what kind of information is being forgotten over time. We found a significant effect of emotion $\left(\mathrm{F}_{(2,72)}=5.00, P=0.011\right.$; Fig. $\left.3 \mathrm{C}\right)$, where emotional stimuli are forgotten more than neutral stimuli (post-hoc Scheffé: $\left.F_{(1,36)}=8.51, P=0.006\right)$. There was a marginally significant effect of memory type $\left(F_{(1,36)}=3.79, P=0.060\right)$, where lure discrimination was worse over time compared to target recognition. Further, we found a significant interaction between emotion and memory type $\left(F_{(2,72)}=9.56, P<0.001\right)$, such that negative lure discrimination was worse $24 \mathrm{~h}$ later compared to immediate performance and other emotion conditions (post-hoc Scheffé: $F_{(1,36)}=18.09, P$ $<0.001)$. This supports the hypothesis that emotional detail information is lost over time while gist information tends to be relatively well preserved.

\section{Applications of the task in cognitively normal aging}

We then applied the task to a sample of 48 cognitively normal older adults (mean age $=76,31 \%$ male). We performed a repeated-measures ANOVA for both target recognition and lure discrimination, with time as the within-subjects factor (immediate and 24-h delay) and age group as the between-subjects factor (young vs. old). For target recognition, we found a main effect of time $\left(F_{(1,83)}=192.81, P<.001\right.$; Fig $\left.4 \mathrm{~A}\right)$, where target recognition was better immediately compared to $24 \mathrm{~h}$ later. We found a main effect of age group $\left(F_{(1,83)}=13.02, P=0.001\right)$, where older adults performed worse than young adults. There was no interaction between age group and time $\left(F_{(1,83)}=0.06, P=0.812\right)$. For lure discrimination, we found a main effect of time $\left(F_{(1,83)}=90.62, P<\right.$ 0.001 ; Fig. $4 \mathrm{~B}$ ), where lure discrimination was better immediately compared to $24 \mathrm{~h}$ later. We found a significant main effect of age group $\left(F_{(1,83)}=6.40, P=0.013\right)$, where older adults performed worse than young adults, and a significant interaction between age group and time $\left(F_{(1,83)}=5.58, P=0.021\right)$, where older adults were significantly worse at discriminating similar lures compared to young adults, but only when tested immediately. When comparing the proportion forgotten across target recognition and lure discrimination across age groups, we found no significant effects of memory type or age ( $P$ 's $>0.05$; Fig. $4 \mathrm{C})$.

Older adults also underwent a neuropsychological testing session that included tests of attention, memory, and executive function. We examined the relationship between our task measures and standard episodic memory tests. We created a composite episodic memory score (see Leal et al. 2018) including the California Verbal Learning Test (immediate and delayed recall) as well as the Weschler Memory Scale-Visual Reproduction Test (immediate and delayed recall) and correlated it to our task measures. We found significant correlations between our composite episodic memory score and immediate target recognition $(r=0.448, P=$ $0.001)$, immediate lure discrimination $(r=0.320, P=0.027)$, and
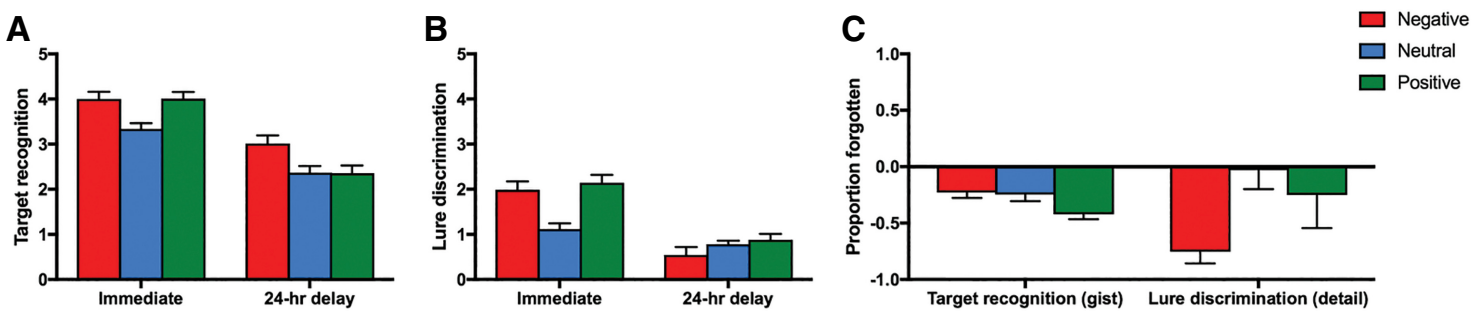

Figure 3. Young adult performance on the video mnemonic discrimination task split by emotion. (A) Target recognition performance split by emotion (negative, neutral, and positive) immediately and $24 \mathrm{~h}$ later in young adults, (B) lure discrimination performance split by emotion immediately and $24 \mathrm{~h}$ later in young adults, (C) proportion forgetting [(Delayed - Immediate)/Immediate] of target and lure stimuli across emotion. 
A
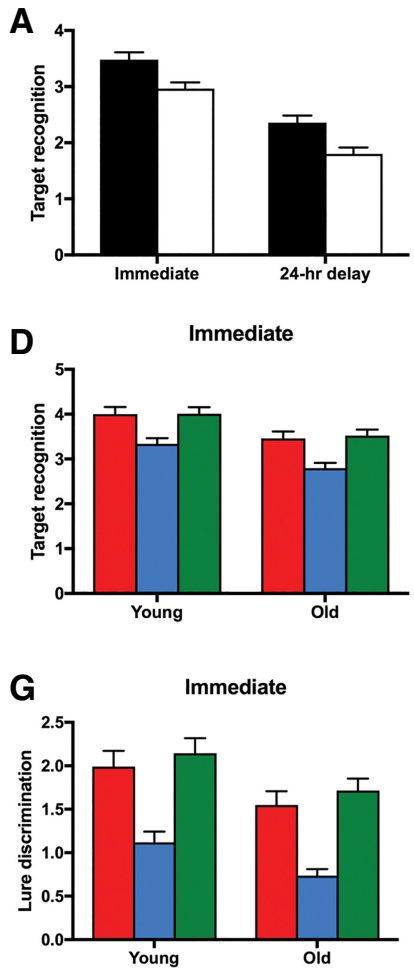

B

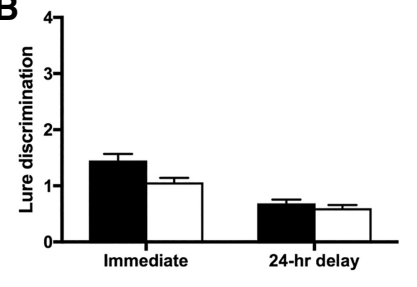

E

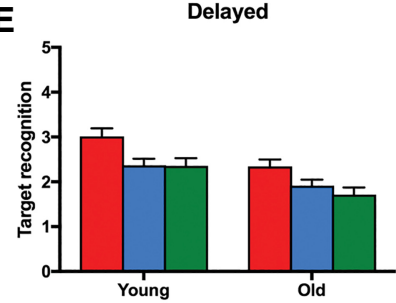

H

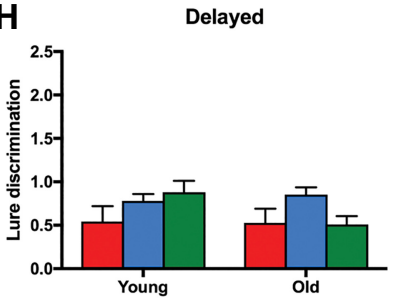

C
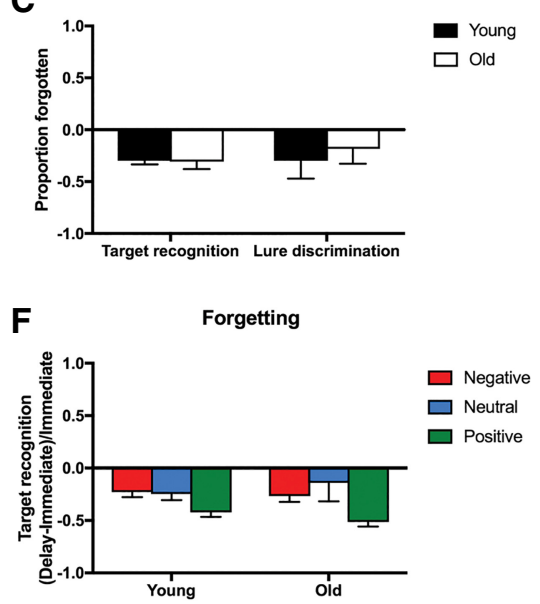

I

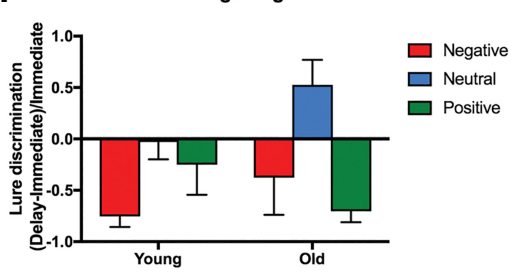

Figure 4. Young and older adult performance on the video mnemonic discrimination task. (A) Overall target recognition performance immediately and $24 \mathrm{~h}$ later in young and older adults, $(B)$ overall lure discrimination performance immediately and $24 \mathrm{~h}$ later in young and older adults, $(C)$ proportion forgetting [(Delayed-Immediate)/Immediate] across memory type and age group, $(D)$ immediate target recognition split by emotion (negative, neutral, and positive) and age (young and old), $(E)$ target recognition after a $24 \mathrm{~h}$ delay split by emotion and age, $(F)$ proportion of targets forgotten split by emotion and age, $(G)$ immediate lure discrimination split by emotion and age, $(H)$ lure discrimination after a $24 \mathrm{~h}$ delay split by emotion and age, $(I)$ proportion of lures forgotten split by emotion and age.

delayed target recognition $(r=0.367, P=0.010)$. We did not find a significant relationship between our composite episodic memory score and delayed lure discrimination $(r=0.180, P=0.221)$, suggesting our delayed lure discrimination measure may be tapping into a different component of memory that is not captured with standard episodic memory tasks.

Next, we divided the stimuli by emotion to test hypotheses on the impact of emotion on memory performance in aging. We conducted a repeated-measures ANOVA with time and emotion as within-subjects factors and age group as a between-subjects factor for both target recognition and lure discrimination. For target recognition, we found a significant effect of time $\left(F_{(1,83)}=261.89, P<\right.$ 0.001 ; Fig. 4D,E), where performance was worse after $24 \mathrm{~h}$. There was a significant main effect of emotion $\left(F_{(2,166)}=26.91, P<\right.$ 0.001 ), where emotional stimuli (both negative and positive) were better remembered than neutral stimuli (post-hoc Scheffé: $\left.F_{(1,83)}=52.52, P<0.001\right)$. We also found a significant interaction between time and emotion $\left(F_{(2,166)}=15.41, P<0.001\right)$, such that emotional targets (both negative and positive) were better remembered immediately compared to neutral targets across age groups while $24 \mathrm{~h}$ later only negative stimuli were better remembered compared to neutral or positive targets across age groups (post-hoc Scheffé: $\left.F_{(1,83)}=16.93, P<0.001\right)$. There was a significant effect of age $\left(F_{(1,83)}=13.92, P<0.001\right)$, where older adults performed worse than young adults, however, there were no interactions between age and any other factor. Thus, target recognition of emotional versus neutral stimuli is similar across age groups, however, older adults have worse performance overall.

Next, we examined the proportion of targets forgotten over time and compared across age groups. We conducted a repeated- measures ANOVA with emotion and age. We found a significant effect of emotion $\left(F_{(2,166)}=5.65, P=0.012\right.$; Fig. $\left.4 \mathrm{~F}\right)$, where positive stimuli were forgotten more than negative and neutral stimuli (post-hoc Scheffé: $\left.F_{(1,83)}=14.52, P<0.001\right)$. There was no significant effect of age or interaction between emotion and age on proportion of target forgetting.

For lure discrimination, we found a significant effect of time $\left(F_{(1,83)}=121.99, P<0.001\right.$; Fig. $\left.4 \mathrm{G}, \mathrm{H}\right)$, where memory for lures was worse after $24 \mathrm{~h}$. There was a significant main effect of emotion overall $\left(F_{(2,166)}=14.92, P<0.001\right)$, where emotional lures were better remembered than neutral lures overall (post-hoc Scheffé: $F_{(1,83)}$ $=37.81, P<0.001)$. We also found a significant interaction between time and emotion $\left(F_{(2,166)}=26.42, P<0.001\right)$, such that emotional lures (both negative and positive) were better remembered immediately compared to neutral lures across age groups while these effects disappear $24 \mathrm{~h}$ later (post-hoc Scheffé: $F_{(1,83)}=91.91, P<$ $0.001)$. There was a significant effect of age $\left(F_{(1,83)}=5.89, P=\right.$ $0.017)$, where older adults performed worse than young adults, and a significant interaction between time and age $\left(F_{(1,83)}=4.05\right.$, $P=0.047)$, where there were larger age differences immediately versus $24 \mathrm{~h}$ later. Interestingly, lure discrimination in older adults showed unique differences when split by emotion after 24 $\mathrm{h}$. Post-hoc testing revealed older adults showed better neutral compared to emotional (both negative and positive) lure discrimination after a 24 h delay (post-hoc Scheffé: $F_{(1,47)}=8.23, P=0.006$ ), while this was not the case for young adults $(P>0.05)$. This suggests that when tested immediately, emotional stimuli are better remembered regardless of age or memory type (i.e., targets or lures), however, after a 24-h delay, this effect is not sustained after a 24-h delay and the pattern reverses with age. 
Next, we examined the proportion of lures forgotten over time and compared across age groups. We conducted a repeatedmeasures ANOVA with emotion and age. We found a significant effect of emotion $\left(F_{(2,166)}=7.87, P=0.001\right.$; Fig. 4I), where emotional lure stimuli (both negative and positive) were forgotten more than neutral lure stimuli (post-hoc Scheffé: $F_{(1,83)}=16.12, P<0.001$ ). There was no significant effect of age or interaction between emotion and age on proportion of lure forgetting.

In order to determine if the type of forgetting differs across memory type (target recognition/gist vs. lure discrimination/detail), we conducted a repeated-measures ANOVA with emotion, memory type, and age. We found a significant effect of emotion $\left(F_{(2,166)}=10.16, P<0.001\right.$; Fig. 4F,I), where emotional stimuli (both negative and positive) were forgotten more than neutral stimuli $\left(F_{(1,83)}=19.23, P<0.001\right)$. We also found a significant interaction between emotion and memory type $\left(F_{(2,166)}=4.74, P=\right.$ $0.014)$, where there was more forgetting of emotional detail information (lure discrimination) compared to emotional gist information (target recognition). This is consistent with previous work suggesting a gist versus detail trade-off (Kensinger et al. 2007; Kensinger 2009; Leal et al. 2014), where emotional gist is relatively preserved at the expense of emotional details.

Older adults also underwent amyloid (Pittsburgh Compound $\mathrm{B}, \mathrm{PIB})$ and tau (Flortaucipir) PET imaging to determine if AD associated pathology was predictive of impaired memory on the task. We split participants based on their global amyloid measure (PIB DVR) into PIB- and PIB+ based on our laboratory threshold of 1.065 PIB DVR. This produced $24 \mathrm{PIB}+$ (mean age $=78,25 \%$ male) and 24 PIB- (mean age $=74,38 \%$ male) participants. Since tau begins to develop in the MTL, we investigated the relationship between MTL tau (including the entorhinal cortex, hippocampus, parahippocampal cortex, and the amygdala [Maass et al. 2018]) and performance on the task in PIB- and PIB+ individuals. We found no significant relationships between MTL tau and target recognition (immediate or delayed) in either PIB+ or PIB- groups ( $P$ 's $>0.025)$. However, we found that MTL tau predicted immediate lure discrimination in PIB+ individuals $(r=-0.492, P=0.014$; Fig. $5 \mathrm{~A})$ and marginally predicted delayed lure discrimination in PIB+ individuals $(r=-0.380, P=0.067$; Fig. 5B). We did not find these same relationships for PIB- individuals $\left(P^{\prime} \mathrm{s}>0.025\right)$. We performed a Fisher r-to-z transform to directly compare the correlation coefficients from the groups for each measure and found a significant difference between PIB+ and PIB- individuals' relationship between MTL tau and immediate lure discrimination $(z=$ $1.94, P=0.052)$ as well as between MTL tau and delayed lure discrimination $(z=1.97, P=0.048)$.

\section{Discussion}

The current study aimed to develop a more ecologically valid memory task using dynamic video stimuli of everyday experiences. Using video clips during encoding allowed participants to encode experiences in a context rather than viewing a context-independent static image, word, or object as is more typically used in memory experiments (Bartels and Zeki 2004; Hasson and Malach 2006; Vanderwal et al. 2017; Güçlütürk et al. 2018). Previously, we developed a mnemonic discrimination task using static scene stimuli (Leal et al. 2014), however, the current mnemonic discrimination task tests memory for dynamic events which has not been done. Within this more naturalistic paradigm, we also parametrically manipulated a variety of factors that impact memory. We included a 24 -h delay memory test since most typical forgetting occurs after $24 \mathrm{~h}$, making delayed testing essential for examining real-world memory. Memory tasks commonly used in neuropsychological batteries and many commonly used memory tasks only test participants within the same day. While this may be easier to conduct experimentally, our memory problems in everyday life result from longer delays between encoding and retrieval.

Another important component to testing real-world memory is the inclusion and evaluation of emotional context, which provides the basis for why we remember certain experiences over others. Many studies either exclude emotion entirely or include emotional components but do not control or evaluate their influence on memory performance. We incorporated video clips of positive, negative, and neutral events one might experience in daily life to capture the varying levels of importance certain experiences might have over others.

Finally, many studies test memory of the exact same items that participants were shown during encoding, however, we often encounter similar but not identical experiences which we must distinguish from previous similar experiences. Inclusion of similar lure stimuli is useful in understanding more specific versus general memory abilities, which may rely on different memory processing mechanisms (i.e., pattern separation and pattern completion). Measuring the effects of varying levels of interference creates a more naturalistic memory paradigm that will allow for more accurate characterization of memory.

To our knowledge, this is the first mnemonic discrimination task to include dynamic videos as stimuli to create a more naturalistic encoding environment. Further, measuring effects of emotion, delayed testing, and similarity on memory allow us to explore the complexities of memory by breaking memory performance down into these well characterized conditions. The results from this study can be summarized by a few core findings. First, this task is sensitive to changes in memory over time as well as differences in the types of memory being measured (i.e., gist versus detail memory). Second, emotion modulates memory differently depending on whether or not consolidation has occurred. While emotion boosts memory performance immediately regardless of memory type, there is a gist versus detail trade-off after $24 \mathrm{~h}$, where negative gist stimuli are relatively preserved over time while emotional detail memory is more forgotten over time. Third, older adults show similar patterns of forgetting emotional versus neutral information over time, however, consolidation of detailed information differs from young adults.

We found that memory for repeated stimuli (target recognition) and the ability to discriminate similar lure stimuli (lure
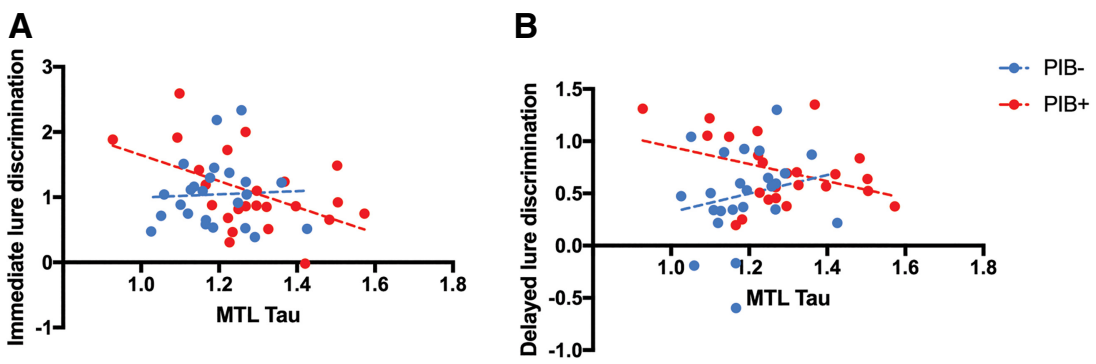

Figure 5. MTL tau predicts lure discrimination in older adults with preclinical Alzheimer's pathologic change. $(A)$ MTL tau (measured via flortaucipir) in cognitively normal older adults predicts immediate lure discrimination in PIB+ (>1.065 PIB DVR threshold) but not in PIB- older adults. (B) MTL tau (measured via flortaucipir) in cognitively normal older adults marginally predicts delayed lure discrimination in PIB+ but not in PIB- older adults. 
discrimination) was impaired after $24 \mathrm{~h}$, however, lure discrimination was worse compared to target recognition. This has been shown in previous work (Stark et al. 2013) and suggests different mechanisms underlie these distinct forms of memory (Kim and Yassa 2013). This difference in memory performance between lure discrimination and target recognition is useful when testing hypotheses about general versus specific (or gist versus detail) memories.

When we split the stimuli by emotion, we found that emotional videos (both positive and negative) were better remembered compared to neutral videos when tested immediately. However, this was not the case after $24 \mathrm{~h}$. For target recognition, negative stimuli were remembered better than neutral or positive stimuli, suggesting a relative preservation of emotional gist information after a delay. It is interesting that we only find a preservation effect for the negative stimuli after $24 \mathrm{~h}$ and not the positive stimuli, even though the positive stimuli are rated at the same arousal level as negative stimuli and are also remembered equally well when tested immediately. One possibility is that positive events are not autobiographical, they are not remembered as well after time passes, however, negative events may be more important to remember and generalize regardless of who the event happened to. This will be an important avenue to explore further in future studies.

For lure discrimination, the beneficial effects of emotion on memory immediately were lost after $24 \mathrm{~h}$. When we examined forgetting across task conditions and memory types, we found that there was more forgetting of negative detail information compared to gist information, which is consistent with an emotional gist versus detail trade-off found in previous studies (Adolphs et al. 2001; Kensinger et al. 2007; Kensinger 2009; Leal et al. 2014) where emotional gist is relatively preserved at the expense of emotional details. It may be more adaptive to retain the bigger picture and forget the minute details of an emotional experience (Loftus et al. 1987).

Another possible explanation for greater forgetting of emotional stimuli over time could be due to an effect of retrievalinduced forgetting (RIF), where retrieval during immediate testing may result in lower recall ability of related items at the second testing timepoint (Anderson et al. 1994). When tested immediately, better memory for emotional items may lead to the reduced ability to recall related (emotional) items later on. Thus, it appears that emotional items are more susceptible to RIF since they are better remembered when tested immediately. It is difficult to distinguish whether the forgetting is caused by a process that actively inhibits information or is due to interference from other related information in memory. Clearly, emotion influences memory differently when tested immediately versus after consolidation has occurred and gives a more accurate picture of real-world memory processing.

We also applied this task to a sample of cognitively normal older adults to test the influence of aging on episodic memory processing. Previous studies have found that mnemonic discrimination is impaired in aging and is a more sensitive measure of memory changes in older adults (Stark et al. 2013). We found that older adults performed worse overall compared to young adults on both memory measures. For target recognition, when we split by emotion, both young and older adults showed a similar pattern of results (better emotional memory immediately and relatively preserved negative memory after $24 \mathrm{~h}$ ). For lure discrimination, we found the age groups did not differ in their ability to perform lure discrimination after $24 \mathrm{~h}$. However, once we split the stimuli by emotion, older adults showed a reversal in emotional mnemonic discrimination after $24 \mathrm{~h}$, such that neutral stimuli were remembered better than positive or negative stimuli. We found a similar effect in a previous study where older adults showed less forgetting of highly similar neutral lures (Leal and Yassa 2014). Young adults showed no difference in lure discrimination across emotion conditions $24 \mathrm{~h}$ later. When examining the proportion of forgetting across memory measures, we found that both age groups showed greater forgetting of emotional detail information compared to gist information, which is consistent with the gist versus detail trade-off found in previous studies (Kensinger 2009; Kensinger et al. 2007; Leal et al. 2014). We showed a similar effect in aging using an emotional mnemonic discrimination task with scenes (Leal and Yassa 2014). Previous studies have shown that older adults may have a bias toward remembering positive information (Mather and Carstensen 2005), however, we did not find evidence of this effect here. Our positive and negative stimuli are matched for arousal, suggesting this is not the underlying cause of the lack of a positivity effect in aging. Again, this may be due to the nature of remembering positive experiences that are not autobiographical, which may influence how experiences are remembered. Our results suggest that including measures of gist versus detail memory, emotion, and time of testing all influence how older adults remember and forget experiences.

Finally, we investigated the influence of amyloid and tau pathology on memory performance to determine if we could detect any relationships between $\mathrm{AD}$ pathology and memory decline early on. Our older participants were all cognitively normal, but half of them were defined as amyloid-positive based on a global PIB threshold (>1.065 PIB DVR). Although we did not formally apply the recent biomarker research framework (Jack et al. 2018), these amyloid positive individuals would be described as older adults with preclinical Alzheimer's pathologic change. We found that older adults on the $\mathrm{AD}$ continuum have more difficulty discriminating highly similar lure items in proportion to the amount of MTL tau. This was not the case for target recognition, suggesting that lure discrimination is a more sensitive measure in detecting early AD pathology. Previous studies have shown a relationship between early amyloid accumulation and tau pathology even in those who are cognitively normal (Leal et al. 2018), suggesting that examining the relationships between memory decline and $\mathrm{AD}$ pathology early on may be beneficial in predicting AD progression. This task may be especially useful early on to detect subtle changes in memory performance.

There are a few limitations of the current study. First, we did not power our sample to test possible sex differences on the task. This could be a fruitful future endeavor, as previous studies have shown sex differences in processing gist versus detail memory (Nielsen et al. 2014). Another possible limitation is that we tested participants on still images from the videos rather than a dynamic clip from the videos. Future studies could consider also testing on video stimuli to determine if inclusion of dynamic stimuli at retrieval changes performance in any way. Further, the effects we find on memory consolidation may be associated with changes in the sleep-wake cycle, where typical findings have shown that older adults have irregular sleep patterns (Buckley and Schatzberg 2005), which may affect their ability to consolidate emotional memories (Payne and Kensinger 2010). This will be an interesting area to explore in future studies. Finally, our participants were pooled from a behavioral study and imaging study, in which the context of performing the experiment in versus out of the scanner could have some influence on performance. Although we did not find any significant differences across groups, having a consistent testing environment is ideal.

Using video stimuli creates a more naturalistic experience for encoding experiences, however, this advantage may not be evident by simply using "Old/New" response options. Future studies using this task along with other existing memory tasks may be able to more directly address various advantages it may have over other versions of mnemonic discrimination tasks (i.e., objects, words, scenes). Participants may show more interest and motivation to participate if the task is more interesting and entertaining 
A novel memory task using naturalistic stimuli

compared to simple objects on a blank screen. Participants may find the video clips more relatable to their own lives and experiences, as we chose videos of events occurring in everyday life, which may influence how participants remember certain videos over others. These will be important aspects to test in the future to determine if using naturalistic video clips to test memory is more ecologically valid than using more simple stimuli (i.e., motivational ratings, task performance differences, etc.). Using more naturalistic tasks versus well-controlled laboratory tasks have different strengths and weaknesses. Memory tasks using simple words or objects are less generalizable to real-world experiences while more naturalistic tasks are complex and more difficult to control, but more closely mimic real-world memory processing.

An important future investigation will be to determine the brain mechanisms underlying these effects. Utilizing a mnemonic discrimination paradigm allows for specific mechanistic hypothesis testing of the hippocampal subfields using high-resolution fMRI and how connectivity between these subfields and the neocortex may change over time (i.e., as a memory becomes consolidated). Most fMRI tasks using mnemonic discrimination paradigms examine brain function during encoding and immediate retrieval, but examining how brain activity and functional connectivity change after a delay has yet to be explored. We hypothesize that the DG/ CA3 subregion of the hippocampus will show a signal consistent with pattern separation (Yassa and Stark 2011) when discriminating similar still images from the videos, especially in the context of emotional stimuli, where the amygdala will likely play a role in modulating this signal (Leal et al. 2014) and has been shown to play an important role in the emotional modulation of memory (McGaugh 2004). Further, we expect that these relationships will shift after $24 \mathrm{~h}$ such that there will likely be more dependence on neocortical regions over time. Future studies examining brain changes over $24 \mathrm{~h}$ will provide clarity as to how memories are consolidated and forgotten over time. Using a more naturalistic task may perhaps activate the MTL and neocortical systems to an even greater extent than using simple objects or words, giving us more signal when examining contrasts between conditions of interest (Bartels and Zeki 2004; Hasson and Malach 2006; Vanderwal et al. 2017; Güçlütürk et al. 2018).

\section{Material and Methods}

\section{Participants}

We recruited 37 young adults from the University of California, Berkeley campus using flyers and email announcements (mean age $=23,27 \%$ male). Twenty-five performed the task in the laboratory and 12 performed the task inside the MRI scanner. We found no significant differences across the different testing environments, so we combined data for a larger sample. We recruited 48 cognitively normal older adults from the Berkeley Aging Cohort Study (BACS; mean age $=76,31 \%$ male). Fifteen performed the task in the laboratory and 33 performed the task inside the MRI scanner. We found no significant differences across the different testing environments, so we combined data for a larger sample. All older adults met the following inclusion criteria: living independently in the community, MMSE $\geq 26$, within age, education, and gender norms on cognitive tests, absence of neurological or psychiatric illness, and lack of major medical illnesses and medications that affect cognition. Demographic information can be found in Table 1.

\section{Neuropsychological testing}

Older participants received a neuropsychological evaluation within three months of participating in the current study. The neuropsychological battery was designed to examine memory
Table 1. Demographic information for young and older adults

\begin{tabular}{lllll}
\hline Variable & Young & Old & PIB- & PIB+ \\
\hline $\mathrm{N}$ & 37 & 48 & 24 & 24 \\
Age & 23 & 76 & 74 & 78 \\
Sex & $10 \mathrm{M}$ & $15 \mathrm{M}$ & $9 \mathrm{M}$ & $6 \mathrm{M}$ \\
Education (yr) & 15 & 17 & 17 & 17 \\
MMSE & - & 29 & 29 & 29 \\
PIB DVR & - & 1.12 & 1.02 & 1.30 \\
MTL Tau SUVR & - & 1.24 & 1.19 & 1.28 \\
\hline
\end{tabular}

Key: MMSE, Mini Mental State Examination; PIB DVR, Pittsburgh Compound B Distributed Volume Ratio; MTL Tau SUVR, Medial temporal lobe tau standardized uptake value ratio.

function, as well as other aspects of general cognitive ability (i.e., the Mini Mental State Examination, MMSE). In particular, the California Verbal Learning Test (CVLT) and the Wechsler Memory Scale-III Visual Reproduction Test (VR) were administered to assess episodic memory processing and to relate to our novel mnemonic discrimination task. We created a composite episodic memory score across these two tests, including both short and long delay intervals. We performed a $Z$-score calculation for each measure using norms from a larger BACS sample $(N=158$, average age $74.1 \pm 5.7 \mathrm{yr}$, average education $16.7 \pm 2.0 \mathrm{yr}, 38 \%$ male).

\section{Amyloid and tau PET imaging}

Older participants also underwent Pittsburgh Compound B (PIB) PET imaging and Flortaucipir (FTP) PET imaging to measure amyloid and tau in the brain, respectively. PET scans occurred within 10 mo of cognitive testing and within 6 mo of task performance. PET scans were acquired on a Siemens Biograph 6 Truepoint PET/ CT scanner in 3D acquisition mode. A low-dose CT scan was collected for attenuation correction. All PIB PET data were preprocessed using Statistical Parametric Mapping (SPM) 12 software (http://www.fil.ion.ucl.ac.uk/spm/), summed, and realigned as previously reported (Leal et al. 2017; Marks et al. 2017). PIB distribution volume ratio (DVR) images were created using Logan graphical analysis with frames corresponding to 35-90 min after injection and a cerebellar gray matter reference region. Mean DVR values from frontal, parietal, temporal, and cingulate cortices were computed to serve as a global PIB index.

FTP standardized uptake value ratio (SUVR) images were created based on mean uptake over 80-100 min postinjection (Baker et al. 2016; Shcherbinin et al. 2016; Wooten et al. 2016) normalized by mean inferior cerebellar gray matter uptake (Maass et al. 2017). Regional analyses based on a priori defined ROIs from a Freesurfer-derived atlas were performed on AV-1451 SUVR images in native space after partial volume correction (PVC) using the Geometric Transfer Matrix approach (Rousset et al. 1998; Desikan et al. 2006; Baker et al. 2017). After PVC and renormalization by the PVC mean of inferior cerebellar gray, we calculated weighted mean SUVR of the MTL (includes the entorhinal cortex, hippocampus, parahippocampal cortex, and the amygdala) since tau in MTL regions, especially the entorhinal cortex, was identified as the best predictor of episodic memory performance (Braak and Braak 1996; Maass et al. 2017, 2018).

\section{Video discrimination task}

The mnemonic discrimination task (Fig. 1) presented video clips during encoding and tested memory for scenes from the video clips immediately and $24 \mathrm{~h}$ later. The stimulus set for encoding was comprised of short video clips acquired from Pond5 (https:// www.pond5.com/), a website that has stock video footage in high definition. The stimulus set for retrieval was comprised of 
A novel memory task using naturalistic stimuli

scenes taken from the $10 \mathrm{~s}$ video, Google searches of similar scenes from the videos, or brand-new images.

An Apple iMac equipped with PsychoPy v1.83.01 was used to present the video clips and scenes as well as record keyboard responses. Each trial consisted of two displays: a video (10 sec) or image (4 sec) display and an ISI display (black screen, $1 \mathrm{sec}$ ). All video clip or scene dimensions were $1140 \times 900$. Video clips were played for $10 \mathrm{sec}$ with an inter-stimulus interval (ISI) of $1 \mathrm{sec}$ with no sound. After $4 \mathrm{sec}$, response options appeared on the screen where participants had to determine whether the videos took place more indoors or more outdoors ( $1=$ INDOOR, $2=$ OUTDOOR). Participants were instructed to respond any time after this point until the video ended at $10 \mathrm{sec}$ and to continue watching the video until it moved on to the next video clip. The experimental paradigm consisted of 120 video clips shown during encoding, split into three 40 video clips blocks ( $7.3 \mathrm{~min}$ each). Half the video clips were neutral and the other half were emotional (positive and negative). Ratings of valence, arousal, and similarity of the videos are described in the next section.

We then tested memory for scenes from the video clips, with half of the scenes tested on Day 1 (about 5 min after the encoding phase) and half the scenes tested $24 \mathrm{~h}$ later on Day 2 . Scenes were randomized across participants. On Day 1, 90 scenes were shown during the immediate test ( $4 \mathrm{sec}$ duration, $1 \mathrm{sec}$ ISI), with one third of the scenes taken directly from the video (targets), one third being similar but not exactly the same (lures), and the remaining third being completely new scenes not shown in any of the video clips (foils). On Day 2, the other half of the stimuli were tested, with the same breakdown of targets, lures, and foils. Participants were instructed to press button 1 if the scene was exactly the same as a scene from one of the video clips they watched or to press button 2 if it was new or different in any way $(1=$ EXACT SAME, $2=$ NEW/DIFFERENT). Response options appeared below each image immediately and participants were instructed to respond while the image was still on the screen. Targets, lures, and foils were evenly distributed across emotion.

Our two key outcome measures were target recognition and lure discrimination. These were measured immediately and $24 \mathrm{~h}$ later. Target recognition was measured by a discriminability index $\left(d^{\prime}\right)$, which was calculated as $z$ (Hits) $-z$ (False Alarms). Hits and false alarms refer to correct recognition of old items and false recognition of new items, respectively. $\mathrm{D}^{\prime}$ is calculated as the difference of $z$-transformed values. In order to measure how well participants discriminated similar items (lures), we examined performance using a discriminability index $\left(\mathrm{d}^{\prime}\right)$, which was calculated as $\mathrm{z}$ (Hits) $-z$ (Lure False Alarms). We also calculated a forgetting rate to determine how much an individual forgets over the 24-h delay, calculated as a proportion of forgetting [(24-h delay $\mathrm{d}^{\prime}$ - Immediate $\left.\mathrm{d}^{\prime}\right)$ / Immediate $\left.d^{\prime}\right]$. These measures were determined overall as well as split into negative, neutral, and positive trials.

\section{Valence, arousal, and similarity measures}

A subset of young participants underwent valence, arousal, and similarity ratings after they completed the study $(N=21$, mean age $=22,33 \%$ male). Participants rated the video clips and images for emotional valence on a scale of 1-9 (1 being the most negative, 9 being the most positive, and 5 being neutral). Video clips and images were also rated for emotional arousal on a scale of 1-9 (1 being the least arousing and 9 being the most arousing). Table 2 shows the average ratings across negative, neutral, and positive stimuli. Negative video clips $\left(t_{(20)}=-15.6, P<0.001\right)$ and images $\left(t_{(20)}=\right.$ $-11.9, P<0.001)$ as well as positive video clips $\left(t_{(20)}=-14.6, P<\right.$ $0.001)$ and images $\left(t_{(20)}=-10.6, P<0.001\right)$ were rated significantly different from neutral stimuli for valence. Both negative video clips $\left(t_{(20)}=9.2, P<0.001\right)$ and images $\left(t_{(20)}=8.6, P<0.001\right)$ as well as
Table 2. Valence and arousal ratings for video clips and images

\begin{tabular}{llll}
\hline Ratings (1-9 scale) & Negative & Neutral & Positive \\
\hline Valence-Videos & $3.3(0.52)$ & $5.4(0.26)$ & $6.7(0.42)$ \\
Valence-Images & $3.6(0.51)$ & $5.3(0.29)$ & $6.7(0.62)$ \\
Arousal-Videos & $5.4(1.1)$ & $3.1(0.63)$ & $5.6(0.76)$ \\
Arousal-Images & $5.2(1.3)$ & $3.0(0.77)$ & $5.6(1.2)$ \\
\hline
\end{tabular}

positive video clips $\left(t_{(20)}=-19.3, P<0.001\right)$ and images $\left(t_{(20)}=\right.$ $-12.3, P<0.001)$ were rated as having higher arousal compared to neutral stimuli.

Finally, similarity ratings were acquired by showing two images side by side. Participants were asked to rate the similarity between the two images ( 1 being completely different, 2 being somewhat similar, 3 being moderately similar, 4 being very similar, and 5 being identical). This task was self-paced. Identical scenes that were paired together received an average rating of 4.9 , lure pairs received an average rating of 3.1, and completely different paired images received a rating of 1.0. Similarity ratings were distributed across the 1-5 scale and similarity ratings across Day 1 and Day 2 testing did not differ $(P=0.99)$.

\section{Statistical analyses}

All statistical analyses were conducted in SPSS v. 25 (IBM Corp., Armonk, NY). Planned comparisons were conducted using repeated-measures ANOVAs and $t$-tests. Post-hoc statistical tests for ANOVAs were corrected for multiple comparisons using Scheffe's correction. All tests used the General Linear Model (ANOVA and correlations). Correlation analyses were corrected for multiple comparisons when necessary using Bonferroni correction. Comparison of correlations was performed using Fisher $r$-to- $z$ transformations. Normality assumptions were investigated using Kolmogorov-Smirnov tests and all distributions investigated did not significantly deviate from the normal distribution. Repeatedmeasures tests were corrected for error nonsphericity using Greenhouse-Geisser correction. Statistical values were considered significant at a final corrected $\alpha$ level of 0.05 (and 0.025 for correlation analyses to correct for multiple comparisons), which appropriately controls for Type I error.

\section{Acknowledgments}

We thank Suzanne Baker, Anne Maass, Taylor Mellinger, Laura Fenton, and Kaitlin Swinnerton for their assistance with data collection and processing. We would also like to thank the participants of the Berkeley Aging Cohort Study who participated in this study. Avid Radiopharmaceuticals enabled use of the $\left[{ }^{18} \mathrm{~F}\right]$ Flortaucipir tracer, but did not provide direct funding and were not involved in data analysis or interpretation. Research reported in this publication was supported by the National Institute of Aging of the National Institutes of Health under Award Numbers F32AG054116 and R01AG034570 and by the Tau Consortium.

\section{References}

Adolphs R, Denburg NL, Tranel D. 2001. The amygdala's role in long-term declarative memory for gist and detail. Behav Neurosci 115: 983-992. doi:10.1037/0735-7044.115.5.983

Adolphs R, Tranel D, Buchanan TW. 2005. Amygdala damage impairs emotional memory for gist but not details of complex stimuli. Nat Neurosci 8: 512-518. doi:10.1038/nn1413

Anderson MC, Bjork RA, Bjork EL. 1994. Remembering can cause forgetting: retrieval dynamics in long-term memory. J Exp Psychol Learn Mem Cogn 20: 1063-1087. doi:10.1037/0278-7393.20.5.1063

Baker SL, Lockhart SN, Price JC, He M, Huesman RH, Schonhaut D, Faria J, Rabinovici G, Jagust WJ. 2016. Reference tissue-based kinetic evaluation 
of 18F-AV-1451 in aging and dementia. J Nucl Med 58: 332-338. doi:10 $.2967 /$ jnumed.116.175273

Baker SL, Maass A, Jagust WJ. 2017. Considerations and code for partial volume correcting [18F]-AV-1451 tau PET data. Data Brief 15: 648-657. doi:10.1016/j.dib.2017.10.024

Bartels A, Zeki S. 2004. Functional brain mapping during free viewing of natural scenes. Hum Brain Mapp 21: 75-85. doi:10.1002/hbm.10153

Braak H, Braak E. 1996. Frequency of stages of Alzheimer-related lesions in different age categories. Neurobiol Aging 18: 351-357. doi:10.1016/ S0197-4580(97)00056-0

Brown R, Kulik J. 1977. Flashbulb memories. Cognition 5: 73-99. doi:10 .1016/0010-0277(77)90018-X

Buckley TM, Schatzberg AF. 2005. Aging and the role of the HPA axis and rhythm in sleep and memory-consolidation. Am J Geriatr Psychiatry 13: 344-352. doi:10.1097/00019442-200505000-00002

Davis H, Ssmall S, Stern Y, Mayeux R, Feldstein S, Keller F. 2003. Acquisition, recall, and forgetting of verbal information in long-term memory by young, middle-aged, and elderly individuals. Cortex 39: 1063-1091. doi:10.1016/S0010-9452(08)70878-5

Desikan RS, Ségonne F, Fischl B, Quinn BT, Dickerson BC, Blacker D, Buckner RL, Dale AM, Maguire RP, Hyman BT, et al. 2006. An automated labeling system for subdividing the human cerebral cortex on MRI scans into gyral based regions of interest. Neuroimage 31: 968-980. doi:10 $.1016 / j$.neuroimage.2006.01.021

Gallagher M, Burwell R, Burchinal M. 1993. Severity of spatial learning impairment in aging: development of a learning index for performance in the Morris water maze. Behav Neurosci 107: 618-626. doi:10.1037/ 0735-7044.107.4.618

Güçlütürk Y, Güçlü U, van Gerven M, van Lier R. 2018. Representations of naturalistic stimulus complexity in early and associative visual and auditory cortices. Sci Rep 8. doi:10.1038/s41598-018-21636-y

Hasson U, Malach R. 2006. Human brain activation during viewing of dynamic natural scenes. Novartis Found Symp 270: 203-216. doi:10 $.1002 / 9780470034989 . \operatorname{ch} 16$

Jack CR, Bennett DA, Blennow K, Carrillo MC, Dunn B, Haeberlein SB, Holtzman DM, Jagust W, Jessen F, Karlawish J, et al. 2018. NIA-AA research framework: toward a biological definition of Alzheimer's disease. Alzheimers Dement 14: 535-562. doi:10.1016/j.jalz.2018.02.018

Kensinger EA. 2009. Remembering the details: effects of emotion. Emot Rev 1: 99-113. doi: $10.1177 / 1754073908100432$

Kensinger EA, Garoff-Eaton RJ, Schacter DL. 2007. Effects of emotion on memory specificity: memory trade-offs elicited by negative visually arousing stimuli. J Mem Lang 56: 575-591. doi:10.1016/j.jml.2006.05 .004

Khan UA, Liu L, Provenzano FA, Berman DE, Profaci CP, Sloan R, Mayeux R, Duff KE, Small SA. 2014. Molecular drivers and cortical spread of lateral entorhinal cortex dysfunction in preclinical Alzheimer's disease. Nat Neurosci 17: 304-311. doi:10.1038/nn.3606

Kim J, Yassa MA. 2013. Assessing recollection and familiarity of similar lures in a behavioral pattern separation task. Hippocampus 23: 287-294. doi:10.1002/hipo.22087

Leal SL, Yassa MA. 2014. Effects of aging on mnemonic discrimination of emotional information. Behav Neurosci 128: 539-547. doi:10.1037/ bne0000011

Leal SL, Yassa MA. 2015. Neurocognitive aging and the hippocampus across species. Trends Neurosci 38: 800-812. doi:10.1016/j.tins.2015.10 .003

Leal SL, Landau SM, Bell RK, Jagust WJ. 2017. Hippocampal activation is associated with longitudinal amyloid accumulation and cognitive decline. Elife 6

Leal SL, Tighe SK, Yassa MA. 2014. Asymmetric effects of emotion on mnemonic interference. Neurobiol Learn Mem 111: 41-48. doi:10.1016/j .nlm.2014.02.013

Leal SL, Lockhart SN, Maass A, Bell RK, Jagust WJ. 2018. Subthreshold amyloid predicts tau deposition in aging. J Neurosci 38: 4482-4489. doi:10.1523/JNEUROSCI.0485-18.2018

Loftus EF, Loftus GR, Messo J. 1987. Some facts about "weapon focus." Law Hum Behav 11: 55-62. doi:10.1007/BF01044839

Ly M, Murray E, Yassa MA. 2013. Perceptual versus conceptual interference and pattern separation of verbal stimuli in young and older adults. Hippocampus 23: 425-430. doi:10.1002/hipo.22110

Maass A, Landau S, Baker SL, Horng A, Lockhart SN, La JR, Rabinovici GD, Jagust WJ. 2017. Comparison of multiple tau-PET measures as biomarkers in aging and Alzheimer's disease. Neuroimage 157: 448-463. doi:10.1016/j.neuroimage.2017.05.058

Maass A, Lockhart SN, Harrison TM, Bell RK, Mellinger T, Swinnerton K, Baker SL, Rabinovici GD, Jagust WJ. 2018. Entorhinal tau pathology, episodic memory decline, and neurodegeneration in aging. J Neurosci 38: 530-543. doi:10.1523/JNEUROSCI.2028-17.2017

Marks SM, Lockhart SN, Baker SL, Jagust WJ. 2017. Tau and $\beta$-amyloid are associated with medial temporal lobe structure, function, and memory encoding in normal aging. J Neurosci 37: 3192-3201.
Marr D. 1971. Simple memory: a theory for archicortex. Philos Trans $R$ Soc Lond B Biol Sci 262: 23-81. doi:10.1098/rstb.1971.0078

Mather M, Carstensen LL. 2005. Aging and motivated cognition: the positivity effect in attention and memory. Trends Cogn Sci 9: 496-502. doi:10.1016/j.tics.2005.08.005

McGaugh JL. 2000. Memory-a century of consolidation. Science 287: 248251. doi:10.1126/science.287.5451.248

McGaugh JL. 2002. Memory consolidation and the amygdala: a systems perspective. Trends Neurosci 25: 456. doi:10.1016/S0166-2236(02) 02211-7

McGaugh JL. 2004. The amygdala modulates the consolidation of memories of emotionally arousing experiences. Annu Rev Neurosci 27: 1-28. doi:10 $.1146 /$ annurev.neuro.27.070203.144157

Murre JMJ, Dros J. 2015. Replication and analysis of Ebbinghaus' forgetting curve. PLoS One 10: e0120644. doi:10.1371/journal.pone .0120644

Nader K, Schafe GE, LeDoux JE. 2000. The labile nature of consolidation theory. Nat Rev Neurosci 1: 216-219. doi:10.1038/35044580

Nielsen SE, Ahmed I, Cahill L. 2014. Postlearning stress differentially affects memory for emotional gist and detail in naturally cycling women and women on hormonal contraceptives. Behav Neurosci 128: 482-493. doi:10.1037/a0036687

Park DC, Royal D, Dudley W, Morrell R. 1988. Forgetting of pictures over a long retention interval in young and older adults. Psychol Aging 3: 9495. doi:10.1037/0882-7974.3.1.94

Payne JD, Kensinger EA. 2010. Sleep's role in the consolidation of emotional episodic memories. Curr Dir Psychol Sci 19: 290-295. doi:10.1177/ 0963721410383978

Reagh ZM, Roberts JM, Ly M, Diprospero N, Murray E, Yassa MA. 2014. Spatial discrimination deficits as a function of mnemonic interference in aged adults with and without memory impairment. Hippocampus 24: 303-314. doi:10.1002/hipo.22224

Reagh ZM, Ho HD, Leal SL, Noche JA, Chun A, Murray EA, Yassa MA. 2016. Greater loss of object than spatial mnemonic discrimination in aged adults. Hippocampus 26: 417-422. doi:10.1002/hipo.22562

Roberts JM, Ly M, Murray E, Yassa MA. 2014. Temporal discrimination deficits as a function of lag interference in older adults. Hippocampus 24: 1189-1196. doi:10.1002/hipo.22303

Robitsek RJ, Fortin NJ, Koh MT, Gallagher M, Eichenbaum H. 2008. Cognitive aging: a common decline of episodic recollection and spatial memory in rats. J Neurosci 28: 8945-8954. doi:10.1523/JNEUROSCI $.1893-08.2008$

Rousset OG, Ma Y, Evans AC. 1998. Correction for partial volume effects in PET: principle and validation. J Nucl Med 39: 904-911.

Ryan L, Cardoza JA, Barense MD, Kawa KH, Wallentin-Flores J, Arnold WT, Alexander GE. 2012. Age-related impairment in a complex object discrimination task that engages perirhinal cortex. Hippocampus 22: 1978-1989. doi:10.1002/hipo.22069

Shapiro M, Olton D. 1994. Hippocampal function and interference. In What are the memory systems of 1994? (ed. DL Schacter and E Tulving), pp. 87-117. MIT Press, Cambridge, Massachusetts.

Shcherbinin S, Schwarz AJ, Joshi A, Navitsky M, Flitter M, Shankle WR, Devous MD, Mintun MA. 2016. Kinetics of the tau PET tracer 18F-AV-1451 (T807) in subjects with normal cognitive function, mild cognitive impairment, and Alzheimer disease. J Nucl Med 57: 15351542. doi:10.2967/jnumed.115.170027

Squire LR, Stark CEL, Clark RE. 2004. The medial temporal lobe. Annu Rev Neurosci 27: 279-306. doi:10.1146/annurev.neuro.27.070203 .144130

Stark SM, Yassa MA, Lacy JW, Stark CEL. 2013. A task to assess behavioral pattern separation (BPS) in humans: data from healthy aging and mild cognitive impairment. Neuropsychologia 51: 2442-2449. doi:10.1016/j neuropsychologia.2012.12.014

Teyler TJ, DiScenna P. 1986. The hippocampal memory indexing theory. Behav Neurosci 100: 147-154. doi:10.1037/0735-7044.100.2.147

Toner CK, Pirogovsky E, Kirwan CB, Gilbert PE. 2009. Visual object pattern separation deficits in nondemented older adults. Learn Mem 16: 338342. doi:10.1101/lm.1315109

Treves A, Rolls ET. 1994. Computational analysis of the role of the hippocampus in memory. Hippocampus 4: 374-391. doi:10.1002/hipo .450040319

Tulving E. 2002. Episodic memory: from mind to brain. Annu Rev Psychol 53: 1-25. doi:10.1146/annurev.psych.53.100901.135114

Vanderwal T, Eilbott J, Finn ES, Craddock RC, Turnbull A, Castellanos FX. 2017. Individual differences in functional connectivity during naturalistic viewing conditions. Neuroimage 157: 521-530. doi:10.1016/ j.neuroimage.2017.06.027

Ward MT, Oler JA, Markus EJ. 1999. Hippocampal dysfunction during aging I: deficits in memory consolidation. Neurobiol Aging 20: 363-372. doi:10 .1016/S0197-4580(99)00045-7

Wilson IA, Ikonen S, Gureviciene I, McMahan RW, Gallagher M, Eichenbaum H, Tanila H. 2004. Cognitive aging and the hippocampus: 
A novel memory task using naturalistic stimuli

how old rats represent new environments. J Neurosci 24: 3870-3878. doi:10.1523/JNEUROSCI.5205-03.2004

Wilson IA, Gallagher M, Eichenbaum H, Tanila H. 2006. Neurocognitive aging: prior memories hinder new hippocampal encoding. Trends Neurosci 29: 662-670. doi:10.1016/j.tins.2006.10.002

Wixted JT. 2004. The psychology and neuroscience of forgetting. Annu Rev Psychol 55: 235-269. doi:10.1146/annurev.psych.55.090902.141555

Wooten D, Guehl NJ, Verwer EE, Shoup TM, Yokell DL, Zubcevik N,

Vasdev N, Zafonte RD, Johnson KA, El Fakhri G, et al. 2016.

Pharmacokinetic evaluation of the tau PET radiotracer [18F]T807 ([18F]

AV-1451) in human subjects. J Nucl Med 58: 484-491. doi:10.2967/ jnumed.115.170910

Yassa MA. 2014. Ground zero in Alzheimer's disease. Nat Neurosci 17: 146147. doi:10.1038/nn.3631
Yassa MA, Stark CEL. 2011. Pattern separation in the hippocampus. Trends Neurosci 34: 515-25. doi:10.1016/j.tins.2011.06.006

Yassa MA, Lacy JW, Stark SM, Albert MS, Gallagher M, Stark CEL. 2011a. Pattern separation deficits associated with increased hippocampal CA3 and dentate gyrus activity in nondemented older adults. Hippocampus 21: 968-979. doi:10.1002/hipo.20808

Yassa MA, Mattfeld AT, Stark SM, Stark CEL. 2011b. Age-related memory deficits linked to circuit-specific disruptions in the hippocampus. Proc Natl Acad Sci 108: 8873-8878. doi:10.1073/pnas.1101567108

Received January 29, 2019; accepted in revised form April 30, 2019. 


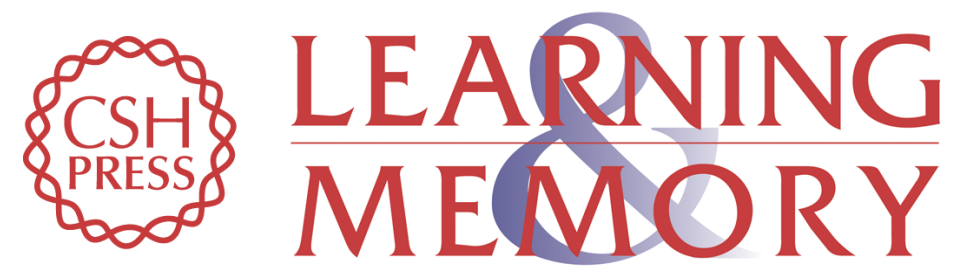

\section{Development of a mnemonic discrimination task using naturalistic stimuli with applications to aging and preclinical Alzheimer's disease}

Stephanie L. Leal, Lorena A. Ferguson, Theresa M. Harrison, et al.

Learn. Mem. 2019, 26:

Access the most recent version at doi:10.1101/lm.048967.118

References This article cites 61 articles, 12 of which can be accessed free at: http://learnmem.cshlp.org/content/26/7/219.full.html\#ref-list-1

Creative This article is distributed exclusively by Cold Spring Harbor Laboratory Press for the Commons first 12 months after the full-issue publication date (see

License http://learnmem.cshlp.org/site/misc/terms.xhtml). After 12 months, it is available under a Creative Commons License (Attribution-NonCommercial 4.0 International), as described at http://creativecommons.org/licenses/by-nc/4.0/.

Email Alerting Receive free email alerts when new articles cite this article - sign up in the box at the Service top right corner of the article or click here. 